\title{
Extragalactic Stellar Astronomy with the Brightest Stars in the Universe
}

\author{
Rolf Kudritzki ${ }^{1}$, Miguel A. Urbaneja ${ }^{2}$, Fabio Bresolin ${ }^{3}$ and Norbert Przybilla ${ }^{4}$ \\ ${ }^{1}$ Institute for Astronomy, University of Hawaii \\ 2680 Woodlawn Drive, Honolulu, HI 96822, USA \\ email: kud@ifa.hawaii.edu \\ 2 Institute for Astronomy, University of Hawaii \\ email: urbaneja@ifa.hawaii.edu \\ ${ }^{3}$ Institute for Astronomy, University of Hawaii \\ email: bresolin@ifa.hawaii.edu \\ ${ }^{4}$ Dr. Remeis-Sternwarte Bamberg, Erlangen University \\ Sternwartstr. 7, D-96049 Bamberg, Germany \\ email: przybilla@sternwarte.uni-erlangen.de
}

\begin{abstract}
A supergiants are objects in transition from the blue to the red (and vice versa) in the uppermost HRD. They are the intrinsically brightest "normal" stars at visual light with absolute visual magnitudes up to -9 . They are ideal to study young stellar populations in galaxies beyond the Local Group to determine chemical composition and evolution, interstellar extinction, reddening laws and distances. We discuss most recent results on the quantitative spectral analysis of such objects in galaxies beyond the Local Group based on medium and low resolution spectra obtained with the ESO VLT and Keck. We describe the analysis method including the determination of metallicity and metallicity gradients. A new method to measure accurate extragalactic distances based on the stellar gravities and effective temperatures is presented, the flux weighted gravity - luminosity relationship (FGLR). The FGLR is a purely spectroscopic method, which overcomes the untertainties introduced by interstellar extinction and variations of metallicity, which plague all photometric stellar distance determination methods. We discuss the perspectives of future work using the giant ground-based telescopes of the next generation such as the TMT, the GMT and the E-ELT.
\end{abstract}

Keywords. galaxies: distances, galaxies: abundances, galaxies: stellar content, galaxies: individual(NGC300), stars: early-type, stars: abundances, stars: distances

\section{Introduction}

It has long been the dream of stellar astronomers to study individual stellar objects in distant galaxies to obtain detailed spectroscopic information about the star formation history and chemodynamical evolution of galaxies and to determine accurate distances based on the determination of stellar parameters and interstellar reddening and extinction. At the first glance, one might think that the most massive and, therefore, most luminous stars with masses higher than 50 $M_{\odot}$ are ideal for this purpose. However, because of their very strong stellar winds and mass-loss these objects keep very hot atmospheric temperatures throughout their life and, thus, waste most of their precious photons in the extreme ultraviolet. As we all know, most of these UV photons are killed by dust absorption in the star forming regions, where these stars are born, and the few which make it to the earth can only be observed with tiny UV telescopes in space such as the HST or FUSE and are not accessible to the giant telescopes on the ground.

Thus, one learns quickly that the most promising objects for such studies are massive stars in a mass range between 15 to $40 M_{\odot}$ in the short-lived evolutionary phase, when they leave the hydrogen main-sequence and cross the HRD in a few thousand years as blue supergiants of late B and early A spectral type. Because of the strongly reduced absolute value of bolometric 
correction when evolving towards smaller temperature these objects increase their brightness in visual light and become the optically brightest "normal" stars in the universe with absolute visual magnitudes up to $M_{V} \cong-9.5$ rivaling with the integrated light brightness of globular clusters and dwarf spheroidal galaxies. These are the ideal stellar objects to obtain accurate quantitative information about galaxies.

\section{Studies in the Milky Way and Local Group}

There has been a long history of quantitative spectroscopic studies of these extreme objects. In a pioneering and comprehensive paper on Deneb Groth (1961) was the first to obtain stellar parameters and detailed chemical composition. This work was continued by Wolf (1971, 1972, 1973) in studies of A supergiants in the Milky Way and the Magellanic Clouds. Kudritzki (1973) using newly developed NLTE model atmospheres found that at the low gravities and the correspondingly low electron densities of these objects effects of departures from LTE can become extremely important. With strongly improved model atmospheres Venn (1995a), Venn (1995b) and Aufdenberg et al. (2002) continued these studies in the Milky Way. Most recently, Przybilla et al. (2006) and Schiller \& Przybilla (2008) used very detailed NLTE line formation calculations including ten thousands of lines in NLTE (see also Przybilla et al. (2000), Przybilla et al. (2001a), Przybilla et al. (2001b), Przybilla \& Butler (2001), Przybilla (2002)) to determine stellar parameters and abundances with hitherto unkown precision $\left(T_{\text {eff }}\right.$ to $\leqslant 2 \%$, $\log g$ to $\sim 0.05 \mathrm{dex}$, individual metal abundances to $\sim 0.05 \mathrm{dex}$ ). At the same time, utelizing the power of the new $8 \mathrm{~m}$ to $10 \mathrm{~m}$ class telescopes, high resolution studies of A supergiants in many Local Group galaxies were carried out by Venn (1999) (SMC), McCarthy et al. (1995) (M33), McCarthy et al. (1997) (M31), Venn et al. (2000) (M31), Venn et al. (2001) (NGC 6822), Venn et al. (2003) (WLM), and Kaufer et al. (2004) (Sextans A) yielding invaluable information about the stellar chemical composition in these galaxies. In the research field of massive stars, these studies have so far provided the most accurate and most comprehensive information about chemical composition and have been used to constrain stellar evolution and the chemical evolution of their host galaxies.

\section{The Challenging Step beyond the Local Group}

The concept to go beyond the Local Group and to study A supergiants by means of quantitative spectroscopy in galaxies out to the Virgo cluster has been first presented by Kudritzki, Lennon \& Puls (1995) and Kudritzki (1998). Following-up on this idea, Bresolin et al. (2001) and Bresolin et al. (2002) used the VLT and FORS at $5 \AA$ resolution for a first investigation of blue supergiants in NGC $3621(6.7 \mathrm{Mpc})$ and NGC $300(1.9 \mathrm{Mpc})$. They were able to demonstrate that for these distances and at this resolution spectra of sufficient $\mathrm{S} / \mathrm{N}$ can be obtained allowing for the quantitative determination of stellar parameters and metallicities. Kudritzki, Bresolin \& Przybilla (2003) extended this work and showed that stellar gravities and temperatures determined from the spectral analysis can be used to determine distances to galaxies by using the correlation between absolute bolometric magnitude and flux weighted gravity $g_{F}=g / T_{e f f}^{4}$ (FGLR). However, while these were encouraging steps towards the use of A supergiants as quantitative diagnostic tools of galaxies beyond the Local Group, the work presented in these papers had still a fundamental deficiency. At the low resolution of $5 \AA$ it is not possible to use ionization equilibria for the determination of $T_{\text {eff }}$ in the same way as in the high resolution work mentioned in the previous paragraph. Instead, spectral types were determined and a simple spectral type - temperature relationship as obtained for the Milky Way was used to determine effective temperatures and then gravities and metallicities. Since the spectral type - $T_{\text {eff }}$ relationship must depend on metallicity (and also gravities), the method becomes inconsistent as soon as the metallicity is significantly different from solar (or 
the gravities are larger than for luminosity class Ia) and may lead to inaccurate stellar parameters. As shown by Evans \& Howarth (2003), the uncertainties introduced in this way could be significant and would make it impossible to use the FGLR for distance determinations. In addition, the metallicities derived might be unreliable. This posed a serious problem for the the low resolution study of A supergiants in distant galaxies.

This problem was overcome only very recently by Kudritzki et al. (2008) (herafter KUBGP), who provided the first self-consistent determination of stellar parameters and metallicities for A supergiants in galaxies beyond the Local Group based on the detailed quantitative model atmosphere analysis of low resolution spectra. They applied their new method on 24 supergiants of spectral type B8 to A5 in the Scultor Group spiral galaxy NGC 300 (at $1.9 \mathrm{Mpc}$ distance) and obtained temperatures, gravities, metallicities, radii, luminosities and masses. The spectroscopic observations were obtained with FORS1 at the ESO VLT in multiobject spectroscopy mode. In addition, ESO/MPI 2.2m WFI and HST/ACS photometry was used. The observations were carried out within the framework of the Araucaria Project (Gieren et al. (2005b)). In the following we discuss the analysis method and the results of this pilot study.

\section{A Pilot Study in NGC300 - Analysis Method}

For the quantitative analysis of the spectra KUBGP use the same combination of line blanketed model atmospheres and very detailed NLTE line formation calculations as Przybilla et al. (2006) in their high signal-to-noise and high spectral resolution study of galactic A-supergiants, which reproduce the observed normalized spectra and the spectral energy distribution, including the Balmer jump, extremely well. They calculate an extensive, comprehensive and dense grid of model atmospheres and NLTE line formation covering the potential full parameter range of all the objects in gravity ( $\log g=0.8$ to 2.5$)$, effective temperature $\left(T_{\text {eff }}=8300\right.$ to $\left.15000 \mathrm{~K}\right)$ and metallicity $\left([Z]=\log Z / Z_{\odot}=-1.30\right.$ to 0.3$)$. The total grid comprises more than 6000 models.

The analysis of the each of the 24 targets in NGC 300 proceeds in three steps. First, the stellar parameters $\left(T_{\text {eff }}\right.$ and $\left.\log g\right)$ are determined together with interstellar reddening and extinction, then the metallicity is determined and finally, assuming a distance to NGC 300, stellar radii, luminosities and masses are obtained. For the first step, a well established method to obtain the stellar parameters of supergiants of late B to early A spectral type is to use ionization equilibria of weak metal lines (OI/II; $\mathrm{MgI} / \mathrm{II} ; \mathrm{NI} / \mathrm{II}$ etc.) for the determination of effective temperature $T_{\text {eff }}$
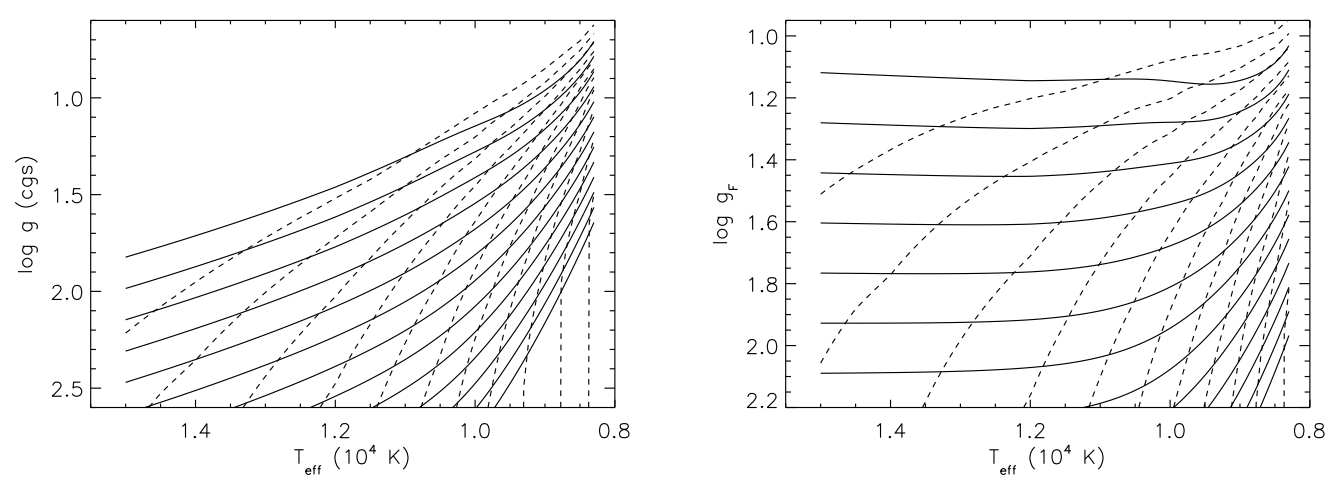

Figure 1. Left:Isocontours of $\mathrm{H} \delta$ equivalent widths (solid) and Balmer jump $D_{B}$ (dashed) in the $\left(\log g, \log T_{e f f}\right)$ plane. $\mathrm{H} \delta$ isocontours start with $1 \AA$ equivalent width and increase in steps of $0.5 \AA$. $D_{B}$ isocontours start with 0.1 dex and increase by 0.1 dex. Right: Same as left but for the flux weighted gravity $\log g_{F}$ instead of gravity $\log g$. Note that this diagram is independent of metallicity, since both the strengths of Balmer lines and the Balmer jump depend only very weakly on metallicity. 
and the Balmer lines for the gravities $\log g$. However, at the low resolution of $5 \AA$ the weak spectral lines of the neutral species disappear in the noise of the spectra and an alternative technique is required to obtain temperature information. KUBGP confirm the result by Evans \& Howarth (2003) that a simple application of a spectral type - effective temperature relationship does not work because of the degeneracy of such a relationship with metallicity. Fortunately, a way out of this dilemma is the use of the spectral energy distributions (SEDs) and here, in particular of the Balmer jump $D_{B}$. While the observed photometry from B-band to I-band is used to constrain the interstellar reddening, $D_{B}$ turns out to be a reliable temperature diagnostic, as is indicated by Fig.1. A simultaneous fit of the Balmer lines and the Balmer jump allows to constrain effective temperature and gravity independent of assumptions on metallicity. Fig.2 demonstrates the sensitivity of the Balmer lines and the Balmer jump to gravity and effective temperature, respectively. At a fixed temperature the Balmer lines allow for a determination of $\log g$ within 0.05 dex uncertainty, whereas the Balmer jump at a fixed gravity yields a temperature uncertainty of 2 percent. However, since the isocontours in Fig.1 are not orthogonal, the maximum errors of $T_{\text {eff }}$ and $\log g$ are 5 percent and 0.2 dex, respectively. These errors are significantly larger than for the analysis of high resolution spectra, but they still allow for an accurate determination of metallicity and distances.

The accurate determination of $T_{e f f}$ and $\log g$ is crucial for the use of A supergiants as distance indicators using the relationship between absolute bolometric magnitude $M_{b o l}$ and flux weighted gravity $\log g_{F}$ defined as

$$
\log g_{F}=\log g-4 \log T_{e f f, 4}
$$

where $T_{\text {eff, } 4}=T_{\text {eff }} / 10000 \mathrm{~K}$ (see Kudritzki, Bresolin \& Przybilla 2003). The relatively large uncertainties obtained with this fit method may casts doubts whether $\log g_{F}$ can be obtained accurately enough. Fortunately, the non-orthogonal behaviour of the fit curves in the left part of Fig.11 leads to errors in $T_{\text {eff }}$ and $\log g$, which are correlated in a way that reduces the uncertainties of $\log g_{F}$. This is demonstrated in right part of Fig.1 which shows the corresponding fit curves of the Balmer lines and $\mathrm{D}_{B}$ in the $\left(\log g_{F}, \log T_{e f f}\right)$ plane. As a consequence, much smaller uncertainties are obtained for $\log g_{F}$, namely \pm 0.10 dex. KUBGP give a detailed discussion of physical reason behind this.
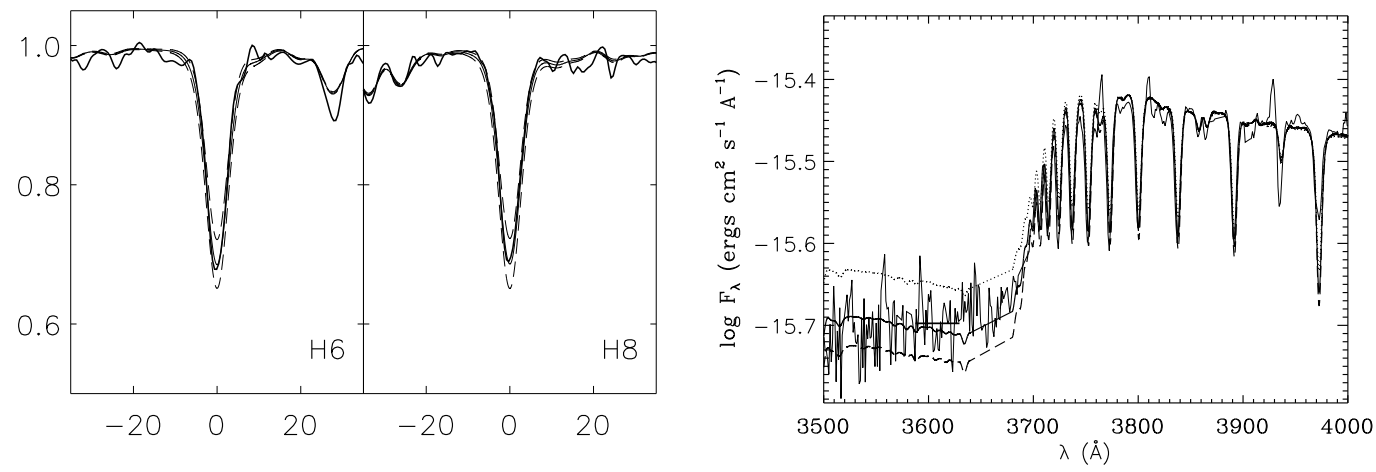

Figure 2. Left: Model atmosphere fit of two observed Balmer lines of NGC300 target No. 21 of KUBGP for $T_{\text {eff }}=10000 \mathrm{~K}$ and $\log g=1.55$ (solid). Two additional models with same $T_{\text {eff }}$ but $\log g=1.45$ and 1.65 , respectively, are also shown (dashed). Right: Model atmosphere fit of the observed Balmer jump of the same target for $T_{\text {eff }}=10000 \mathrm{~K}$ and $\log g=1.55$ (solid). Two additional models with the same $\log g$ but $T_{\text {eff }}=$ $9750 \mathrm{~K}$ (dashed) and $10500 \mathrm{~K}$ (dotted) are also shown. The horizontal bar at $3600 \AA$ represents the average of the flux logarithm over this wavelength interval, which is used to measure $\mathrm{D}_{B}$. 
Knowing the stellar atmospheric parameters $T_{\text {eff }}$ and $\log g$ KUBGP are able to determine stellar metallicities by fitting the metal lines with their comprehensive grid of line formation calculations. The fit procedure proceeds in several steps. First, spectral windows are defined, for which a good definition of the continuum is possible and which are relatively undisturbed by flaws in the spectrum (for instance caused by cosmic events) or interstellar emission and absorption. A typical spectral window used for all targets is the wavelength interval $4497 \AA \leqslant \lambda \leqslant 4607 \AA$. Fig. 3 shows the synthetic spectrum calculated for the atmopsheric parameters of target No. 21 (the previous example) and for all the metallicities of the grid ranging from $-1.30 \leqslant[\mathrm{Z}] \leqslant 0.30$. It is very obvious that the strengths of the metal line features are a strong function of metallicity. In Fig. 4 the observed spectrum of target No. 21 in this spectral region is shown overplotted by the synthetic spectrum for each metallicity. Separate plots are used for each metallicity, because the optimal relative normalization of the observed and calculated spectra is obviously metallicity dependent. This problem is addressed by renormalizing the observed spectrum for each metallicity so that the synthetic spectrum always intersects the observations at the same value at the two edges of the spectral window (indicated by the dashed vertical lines). The next step is a pixel-by-pixel comparison of calculated and normalized observed fluxes for each metallicity and a calculation of a $\chi^{2}$-value. The minimum $\chi([Z])$ as a function of $[Z]$ is then used to determine the metallicity. This is also shown in Fig.4 Application of the same method on different spectral windows provides additional independent information on metallicity and allows to determine the average metallicity obtained from all windows. A value of is $[\mathrm{Z}]=-0.39$ with a very small dispersion of only 0.02 dex. However, one also need to consider the effects of the stellar parameter uncertainties on the metallicity determination. This is done by applying the same correlation method for [Z] for models at the extremes of the error box for $T_{\text {eff }}$ and $\log g$. This increases the uncertainty of $[\mathrm{Z}]$ to $\pm 0.15 \mathrm{dex}$, still a very reasonable accuracy of the abundance determination.

The fit of the observed photometric fluxes with the model atmosphere fluxes was used to determine interstellar reddening $\mathrm{E}(\mathrm{B}-\mathrm{V})$ and extinction $\mathrm{A}_{V}=3.1 \mathrm{E}(\mathrm{B}-\mathrm{V})$. Simultaneously, the fit also yields the stellar angular diameter, which provides the stellar radius, if a distance is adopted. Alternatively, for the stellar parameters $\left(T_{e f f}, \log g,[Z]\right)$ determined through the spectral analysis the model atmospheres also yield bolometric corrections $\mathrm{BC}$, which we use to determine bolometric magnitudes. These bolometric magnitudes then also allow us to compute radii. The radii determined with these two different methods agree within a few percent. Gieren et al. (2005a)

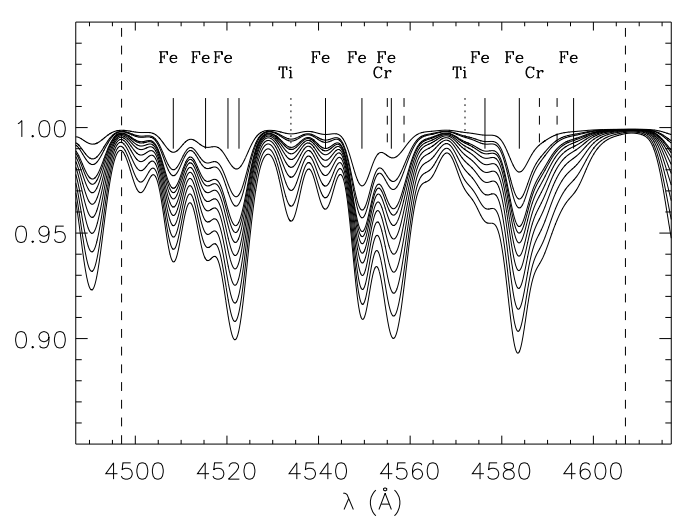

Figure 3. Synthetic metal line spectra calculated for the stellar parameters of target No.21 as a function of metallicity in the spectral window from $4497 \AA$ to $4607 \AA$. Metallicities range from [Z] $=-1.30$ to 0.30 , as described in the text. The dashed vertical lines give the edges of the spectral window as used for a determination of metallicity. 
in their multi-wavelength study of a large sample of Cepheids in NGC 300 including the near-IR have determined a new distance modulus $\mathrm{m}-\mathrm{M}=26.37 \mathrm{mag}$, which corresponds to a distance of $1.88 \mathrm{Mpc}$. KUBGP have adopted these values to obtain the radii and absolute magnitudes.

\section{A Pilot Study in NGC300 - Results}

As a first result, the quantitative spectroscopic method yields interstellar reddening and extinction as a by-product of the analysis process. For objects embedded in the dusty disk of a star forming spiral galaxy one expects a wide range of interstellar reddening $\mathrm{E}(\mathrm{B}-\mathrm{V})$ and, indeed, a range from $\mathrm{E}(\mathrm{B}-\mathrm{V})=0.07 \mathrm{mag}$ up to $0.24 \mathrm{mag}$ was found. The individual reddening values are significantly larger than the value of $0.03 \mathrm{mag}$ adopted in the HST distance scale key project study of Cepheids by Freedman et al. (2001) and demonstrate the need for a reliable reddening determination for stellar distance indicators, at least as long the study is restricted to optical wavelengths. The average over the observed sample is $\langle E(B-V)\rangle=0.12$ mag in close agreement with the value of $0.1 \mathrm{mag}$ found by Gieren et al. (2005a) in their optical to near-IR study of Cepheids in NGC 300. While Cepheids have somewhat lower masses than the A supergiants of our study and are consequently somewhat older, they nonetheless belong to the same population and are found at similar sites. Thus, one expects them to be affected by interstellar reddening in the same way as A supergiants.

Fig.5 shows the location of all the observed targets in the $\left(\log g, \log T_{\text {eff }}\right)$ plane and in the HRD compared to the early B-supergiants studied by Urbaneja et al. (2005). The comparison with evolutionary tracks gives a first indication of the stellar masses in a range from $10 \mathrm{M}_{\odot}$ to 40 $\mathbf{M}_{\odot}$. Three targets have obviously higher masses than the rest of the sample and seem to be on a similar evolutionary track as the objects studied by Urbaneja et al. (2005). The evolutionary information obtained from the two diagrams appears to be consistent. The B-supergiants seem to be more massive than most of the A supergiants. The same three A supergiants apparently more massive than the rest because of their lower gravities are also the most luminous objects. This confirms that quantitative spectroscopy is -at least qualitatively - capable to retrieve the information about absolute luminosities. Note that the fact that all the B supergiants studied by Urbaneja et al. (2005) are more massive is simply a selection effect of the V magnitude limited spectroscopic survey by Bresolin et al. (2002). At similar V magnitude as the A supergiants those objects have higher bolometric corrections because of their higher effective temperatures and are, therefore, more luminous and massive.

Fig.6 shows the stellar metallicities and the metallicity gradient as a function of angular galac-
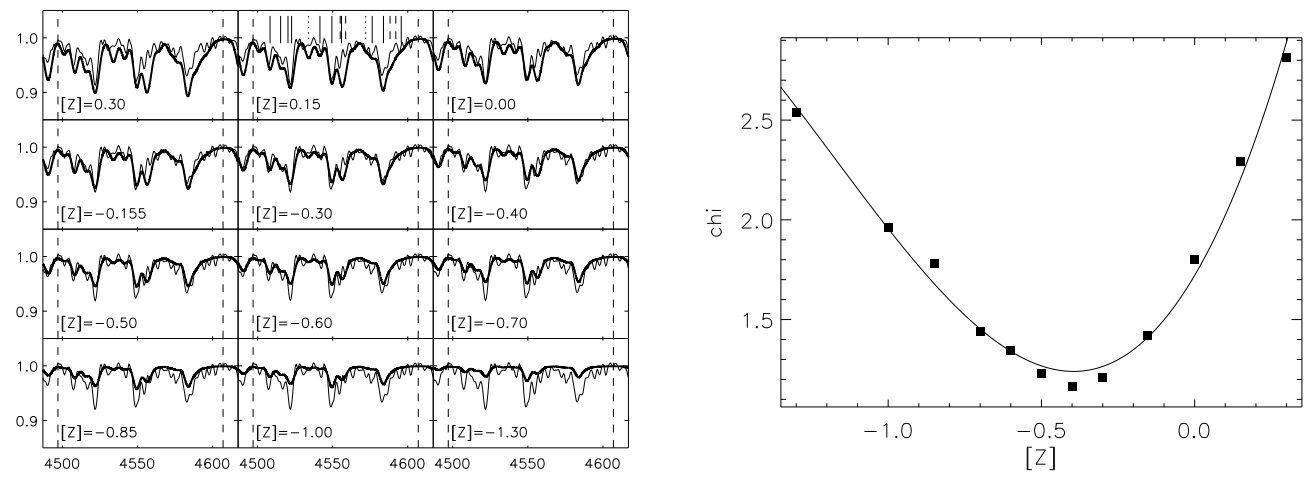

Figure 4. Left: Observed spectrum of target No. 21 for the same spectral window as Fig. 3 overplotted by the same synthetic spectra for each metallicity separately. Right: $\chi([Z])$ as obtained from the comparison of observed and calculated spectra. The solid curve is a third order polynomial fit. 
tocentric distance, expressed in terms of the isophotal radius, $\rho / \rho_{0}$ ( $\rho_{0}$ corresponds to $5.33 \mathrm{kpc}$ ). Despite the scatter caused by the metallicity uncertainties of the individual stars the metallicity gradient of the young disk population in NGC 300 is very clearly visible. A linear regression for the combined A- and B-supergiant sample yields ( $\mathrm{d}$ in $\mathrm{kpc}$ )

$$
[Z]=-0.06 \pm 0.09-(0.083 \pm 0.022) d .
$$

Note that the metallicities of the B supergiants refer to oxygen only with a value of log $\mathrm{N}(\mathrm{O}) / \mathrm{N}(\mathrm{H})=-3.31$ adopted for the sun (Allende Prieto et al. (2001)). On the other hand, the A supergiant metallicities reflect the abundances of a variety of heavy elements such as $\mathrm{Ti}, \mathrm{Fe}$, $\mathrm{Cr}, \mathrm{Si}, \mathrm{S}$, and $\mathrm{Mg}$.

With these results KUBGP extended the discussion started by Urbaneja et al. (2005) to compare with oxygen abundances obtained from HII-region emission lines. Urbaneja et al. (2005) used line fluxes published by Deharveng et al. (1988) and applied various different published strong line method calibrations to determine nebular oxygen abundances, which could then be used to obtain the similar regressions as above.

The different strong line method calibrations lead to significant differences in the central metallicity as well as in the abundance gradient. The calibrations by Dopita \& Evans. (1986) and Zaritsky et al. (1994) predict a metallicity significantly supersolar in the center of NGC 300 contrary to the other calibrations. On the other hand, the work by KUBGP yields a central metallicity slightly smaller than solar in good agreement with Denicolo et al. (2002) and marginally agreeing with Kobulnicky et al. (1999), Pilyugin (2001), and Pettini \& Pagel (2004). At the isophotal radius, $5.3 \mathrm{kpc}$ away from the center of NGC 300, they obtain an average metallicity significantly smaller than solar $[\mathrm{Z}]=-0.50$, close to the average metallicity in the SMC. The calibrations by Dopita \& Evans. (1986), Zaritsky et al. (1994), Kobulnicky et al. (1999) do not reach these small values for oxygen in the HII regions either because their central metallicity values are too high or the metallicity are gradients too shallow.

In light of the substantial range of metallicies obtained from HII region emission lines using different strong line method calibrations it seems to be extremely valuable to have an independent method using young stars. It will be very important to compare our results with advanced work on
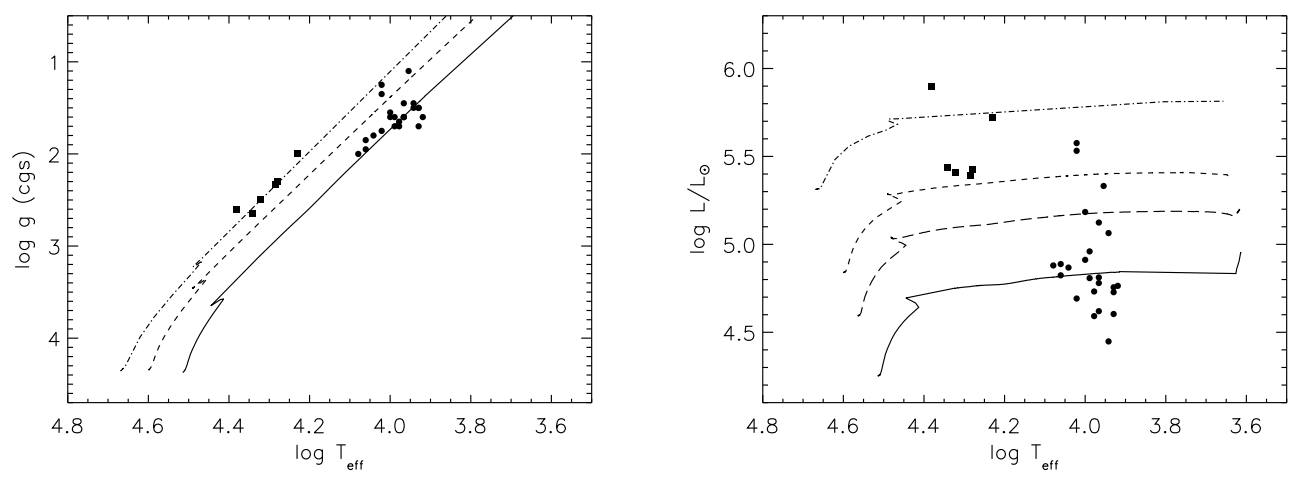

Figure 5. Left: NGC 300 A supergiants (filled circles) and early B supergiants (filled squares) in the $\left(\log g, \log T_{e f f}\right)$ plane compared with evolutionary tracks by Meynet \& Maeder (2005) of stars with 15 $\mathrm{M}_{\odot}$ (solid), $25 \mathrm{M}_{\odot}$ (dashed), and $40 \mathrm{M}_{\odot}$ (dashed-dotted), respectively. Right: NGC $300 \mathrm{~A}$ and early B supergiants in the HRD compared with evolutionary tracks for stars with $15 \mathrm{M}_{\odot}$ (solid), $20 \mathrm{M}_{\odot}$ (longdashed), $25 \mathrm{M}_{\odot}$ (short-dashed), and $40 \mathrm{M}_{\odot}$ (dashed-dotted), respectively. The tracks include the effects of rotation and are calculated for SMC metallicity (see Meynet \& Maeder 2005). 
HII regions, which will avoid strong line methods, and will use direct infomation about nebular electron temperatures and densities (Bresolin et al., 2008, in preparation, see also this volume).

KUBGP discuss the few outliers in Fig.6 and claim that these metallicities seem to be real. Their argument is that the expectation of homogeneous azimuthal metallicity in patchy star forming galaxies seems to be naive. Future work on other galaxies will show whether cases like this are common or not.

\section{Flux Weighted Gravity - Luminosity Relationship (FGLR)}

Massive stars with masses in the range from $12 \mathrm{M}_{\odot}$ to $40 \mathrm{M}_{\odot}$ evolve through the B and A supergiant stage at roughly constant luminosity (see Fig.57). In addition, since the evolutionary timescale is very short when crossing through the B and A supergiant domain, the amount of mass lost in this stage is small. This means that the evolution proceeds at constant mass and constant luminosity. This has a very simple, but very important consequence for the relationship of gravity and effective temperature along each evolutionary track. From

$$
L \propto R^{2} T_{\mathrm{eff}}^{4}=\text { const.; } M=\text { const. }
$$

follows immediately that

$$
M \propto g R^{2} \propto L\left(g / T_{\text {eff }}^{4}\right)=L g_{F}=\text { const. }
$$

Thus, along the evolution through the B and A supergiant domain the "flux-weighted gravity" $g_{F}=g / T_{e f f}^{4}$ should remain constant. This means each evolutionary track of different luminosity in this domain is characterized by a specific value of $g_{F}$. This value is determined by the relationship between stellar mass and luminosity, which in a first approximation is a power law

$$
L \propto M^{x}
$$

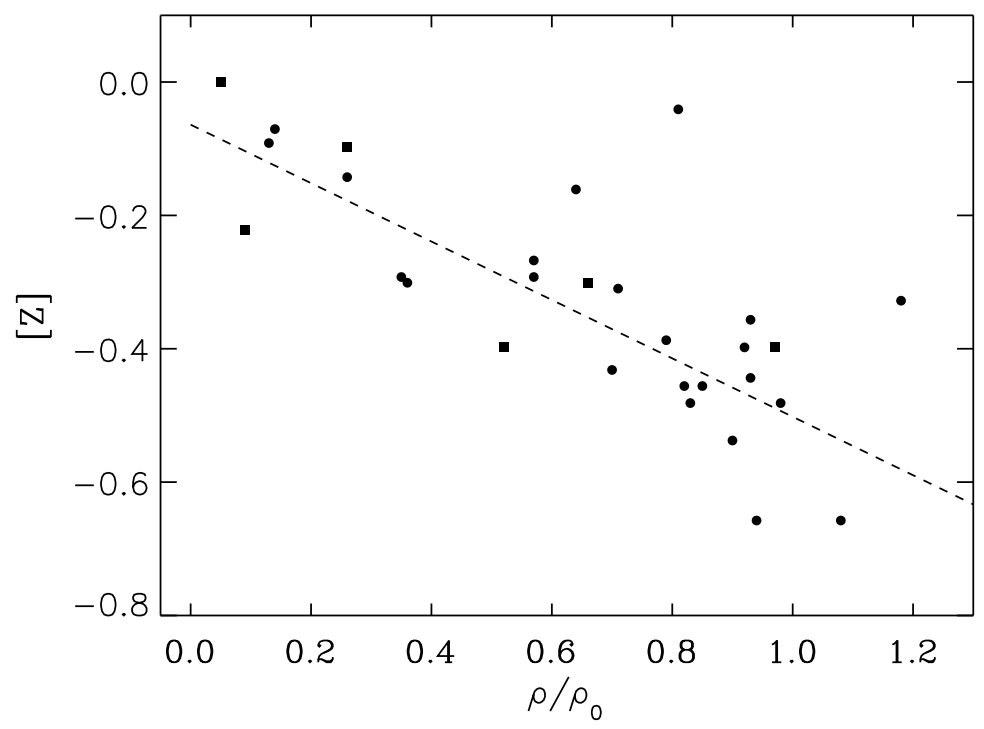

Figure 6. Metallicity [Z] as a function of angular galacto-centric distance $\rho / \rho_{0}$ for the A supergiants (filled circles) and the early B-supergiants studied by Urbaneja et al. (2005) (filled squares). Note that for the latter metallicity refers to oxygen only. The dashed curve represents the regression discussed in the text. 
and leads to a realtionship between luminosity and flux-weighted gravity

$$
L^{1-x} \propto\left(g / T_{\mathrm{eff}}^{4}\right)^{x}
$$

With the definition of bolometric magnitude $M_{\mathrm{bol}} \propto-2.5 \log L$ one then derives

$$
\left.-M_{\mathrm{bol}}=a_{F G L R}\left(\log g_{F}-1.5\right)\right)+b_{F G L R} .
$$

This is the "flux-weighted gravity - luminosity relationship" (FGLR) of blue supergiants. Note that the proportionality constant $a_{F G L R}$ is given by the exponent of the mass - luminosity power law through

$$
a_{F G L R}=2.5 x /(1-x),
$$

for instance, for $x=3$, one obtains $a_{F G L R}=-3.75$. Note that the zero point of the relationship is chosen at a flux weighted gravity of 1.5 , which is in the middle of the range encountered for blue supergiant stars.

KUBGP use the mass-luminosity relationships of different evolutionary tracks (with and without rotation, for Milky Way and SMC metallicity) to calculate the different FGLRs predicted by stellar evolution. Very interestingly, while different evolutionary model types yield somewhat different FGLRs, the differences are rather small.

Kudritzki, Bresolin \& Przybilla (2003) were the first to realize that the FGLR has a very interesting potential as a purely spectroscopic distance indicator, as it relates two spectroscopically well defined quantitities, effective temperature and gravity, to the absolute magnitude. Compiling a large data set of spectroscopic high resolution studies of A supergiants in the Local Group and with an approximate analysis of low resolution data of a few targets in galaxies beyond the Local Group (see discussion in previous chapters) they were able to prove the existence of an observational FGLR rather similar to the theoretically predicted one.

With the improved analysis technique of low resolution spectra of A supergiants and with the much larger sample studied for NGC 300 KUBGP resumed the investigation of the FGLR.

The result is shown in Fig.7, which for NGC 300 (left diagram) reveals a clear and rather tight relationship of flux weighted gravity $\log g_{F}$ with bolometric magnitude $M_{b o l}$. A simple linear regression yields $b_{F G L R}=8.11$ for the zero point and $a_{F G L R}=-3.52$ for the slope. The standard
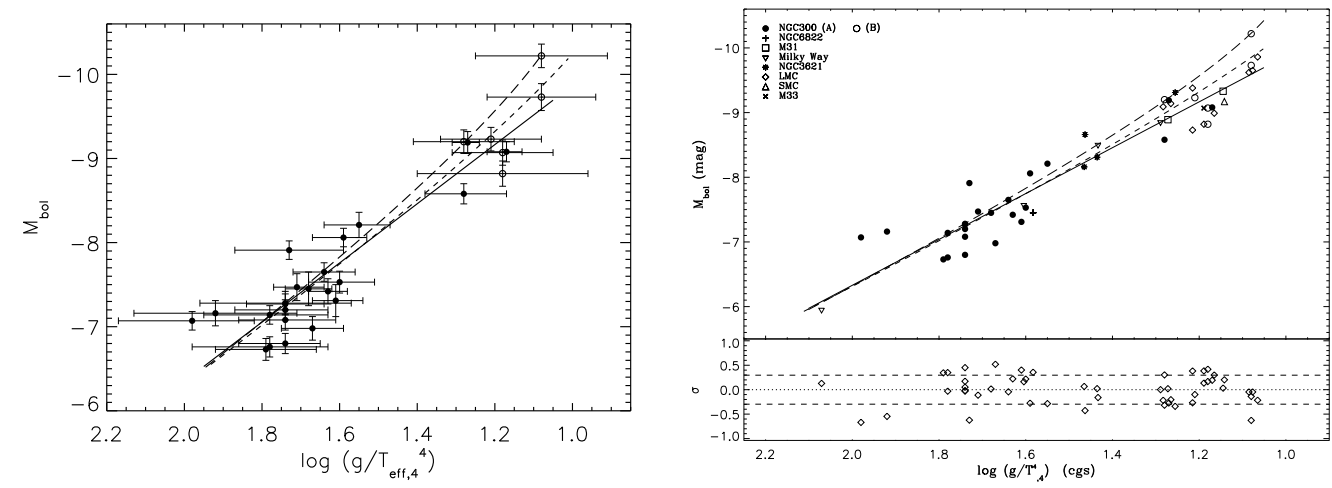

Figure 7. Left: The FGLR of A (solid circles) and B (open circles) supergiants in NGC 300 and the linear regression (solid). The stellar evolution FGLRs for models with rotation are also overplotted (dashed: Milky Way metallicity, long-dashed: SMC metallicity). Right: Same as left, but with additional objects from 7 other galaxies (see text). 
deviation from this relationship is $\sigma=0.34$ mag. Within the uncertainties the observed FGLR appears to be in agreement with the theory.

In their first investigation of the empirical FGLR Kudritzki, Bresolin \& Przybilla (2003) have added A supergiants from six Local Group galaxies with stellar parameters obtained from quantitative studies of high resolution spectra (Milky Way, LMC, SMC, M31, M33, NGC 6822) to their results for NGC 300 to obtain a larger sample. They also added 4 objects from the spiral galaxy NGC 3621 (at $6.7 \mathrm{Mpc}$ ) which were studied at low resolution. KUBGP added exactly the same data set to their new enlarged NGC 300 sample, however, with a few minor modifications. For the Milky Way they included the latest results from Przybilla et al. (2006) and Schiller \& Przybilla (2008) and for the two objects in M31 we use the new stellar parameters obtained by Przybilla et al. (2008). For the objects in NGC 3621 they applied new HST photometry. They also re-analyzed the LMC objects using ionization equilibria for the temperature determination.

Fig.7 (right diagram) shows bolometric magnitudes and flux-weighted gravities for this full sample of eight galaxies revealing a tight relationship over one order of magnitude in fluxweighted gravity. The linear regression coefficients are $a_{F G L R}=-3.41 \pm 0.16$ and $b_{F G L R}=$ $8.02 \pm 0.04$, very simimilar to the NGC 300 sample alone. The standard deviation is $\sigma=0.32$ mag. The stellar evolution FGLR for Milky Way metallicity provides a fit of almost similar quality with a standard deviation of $\sigma=0.31 \mathrm{mag}$.

\section{Conclusions and Future Work}

The astrophysical potential of low resolution spectroscopy of A supergiant stars for studying galaxies beyond the Local Group is quite remarkable. By introducing a novel method for the quantitative spectral analysis one is able to determine accurate stellar parameters, which allow for a detailed test of stellar evolution models. Through the spectroscopic determination of stellar parameters one can also constrain interstellar reddening and extinction by comparing the calculated SED with broad band photometry. The study of NGC 300 finds a very patchy extintion pattern as to be expected for a star forming spiral galaxy. The average extinction is in agreement with multi-wavelength studies of Cepheids including K-band photometry.

The method also allows to determine stellar metallicities and to study stellar metallicity gradients. Solar metallicity is found in the center of NGC 300 and a gradient of $-0.08 \mathrm{dex} / \mathrm{kpc}$. To our knowledge this is the first systematic stellar metallicity study in galaxies beyond the Local Group focussing on iron group elements. In the future the method can be extended to not only determine metallicity but also the ratio of $\alpha$ - to iron group elements as a function of galactocentric distance. The stellar metallicities obtained can be compared with oxygen abundance studies of HII regions using the strong line method. This allows to discuss the various calibrations of the strong line method, which usually yield very different results.

The improved spectral diagnostic method makes it possible to very accurately determine stellar flux weighted gravities $\log g_{F}=\log g / T_{\text {eff }}^{4}$ and bolometric magnitudes. Above a certain threshold in effective temperature a simple measurement of the strengths of the Balmer lines can be used to determine accurate values of $\log g_{F}$.

Absolute bolometric magnitudes $M_{b o l}$ and flux-weighted gravities $\log g_{F}$ are tightly correlated. It is shown that such a correlation is expected for stars, which evolve at constant luminosity and mass. This "flux-weighted gravity - luminosity relationship (FGLR)" agreement with stellar evolution theory.

With a relatively small residual scatter of $\sigma=0.3$ mag the observed FGLR is an excellent tool to determine accurate spectroscopic distance to galaxies. It requires multicolor photometry and low resolution $(5 \AA)$ spectroscopy to determine effective temperature and gravity and, thus, flux-weighed gravity directly from the spectrum. With effective temperature, gravity and metal- 
licity determined one also knows the bolometric correction, which is small for A supergiants, which means that errors in the stellar parameters do not largely affect the determination of bolometric magnitudes. Moreover, one knows the intrinsic stellar SED and, therefore, can determine interstellar reddening and extinction from the multicolor photometry, which then allows for the accurate determination of the reddening-free apparent bolometric magnitude. The application of the FGLR then yields absolute magnitudes and, thus, the distance modulus. With the intrinsic scatter of $\sigma=0.3 \mathrm{mag}$ and 30 targets per galaxy one can estimate an accuracy of 0.05 mag in distance modulus ( $0.1 \mathrm{mag}$ for 10 target stars). The results for WLM by Miguel Urbaneja et al. and for M33 by Vivian $\mathrm{U}$ et al. presented in these proceedings are a first demonstration of the power of the method.

The advantage of the FGLR method for distance determinations is its spectroscopic nature, which provides significantly more information about the physical status of the objects used for the distance determination than simple photometry methods. Most importantly, metallicity and interstellar extinction can be determined directly. The latter is crucial for spiral and irregular galaxies because of the intrinsic patchiness of reddening and extinction.

Since supergiant stars are known to show intrinsic photometric variability, the question arises whether the FGLR method is affected by such variability. For the targets in NGC 300 this issue has been carefully investigated by Bresolin et al. (2004), who studied CCD photometry lightcurves obtained over many epochs in the parallel search for Cepheids in NGC 300. They concluded that amplitudes of photometric variability are very small and do not affect distance determinations using the FGLR method.

The effects of crowding and stellar multiplicity are also important. However, in this regard A supergiants offer tremendous advantages relative to other stellar distance indicators. First of all, they are significantly brighter. Bresolin et al. (2005) using HST ACS photometry compared to ground-based photometry have studied the effects of crowding on the Cepheid distance to NGC 300 and concluded that they are negligible. With A supergiants being 3 to 6 magnitudes brighter than Cepheids it is clear that even with ground-based photometry only crowding is generally not an issue for these objects at the distance of NGC 300 and, of course, with HST photometry (and in the future JWST) one can reach much larger distances before crowding becomes important. In addition, any significant contribution by additional objects to the light of an A supergiant will become apparent in the spectrum, if the contaminators are not of a very similar spectral type, which is very unlikely because of the short evolutionary lifetime in the A supergiant stage. It is also important to note that A supergiants have evolutionary ages larger than 10 million years, which means that they have time to migrate into the field or that they are found in older clusters, which are usually less concentrated than the very young OB associations.

It is evident that the type of work described in this paper can be in a straightforward way extended to the many spiral galaxies in the local volume at distances in the 4 to $7 \mathrm{Mpc}$ range. Pushing the method we estimate that with present day $8 \mathrm{~m}$ to $10 \mathrm{~m}$ class telescopes and the existing very efficient multi-object spectrographs one can reach down with sufficient $\mathrm{S} / \mathrm{N}$ to $\mathrm{V}=22.5$ mag in two nights of observing time under very good conditions. For objects brighter than $M_{V}$ $=-8$ mag this means metallicities and distances can be determined out to distances of $12 \mathrm{Mpc}$ ( $\mathrm{m}-\mathrm{M}=30.5 \mathrm{mag}$ ). This opens up a substantial volume of the local universe for metallicity and galactic evolution studies and independent distance determinations complementary to the existing methods. With the next generation of extremely large telescopes such as the TMT, GMT or the E-ELT the limiting magnitude can be pushed to $\mathrm{V}=24.5$ equivalent to distances of 30 $\mathrm{Mpc}(\mathrm{m}-\mathrm{M}=32.5 \mathrm{mag})$.

\section{References}

Allende Prieto, C., Lambert, D.L., \& Asplund, M. 2001, ApJ, 556, L63 
Aufdenberg, J. P., Hauschildt, P.H., Baron, E. et al. 2002, ApJ, 570, 344

Bresolin, F., Kudritzki, R.-P., Méndez, R. H., \& Przybilla, N. 2001, ApJ, 548, L159

Bresolin, F., Gieren, W., Kudritzki, R.-P., Pietrzyński, G., \& Przybilla, N. 2002, ApJ, 567, 277

Bresolin, F., Pietrzyński, G., Gieren, W., Kudritzki, R.-P., Przybilla, N. \& Fouque, P. 2004, ApJ, 600, 182

Bresolin, F., Pietrzyński, G., Gieren, W. \& Kudritzki, R. P. 2005, ApJ, 634, 1020

Deharveng, L., Caplan, J., Lequeux, J., Azzopardi, M., Breysacher, J., Tarenghi, M., Westerlund, B. 1988, $A \& A S, 73,407$

Denicolo, G., Terlevich, R.\& Terlevich, E. 2002, MNRAS, 330, 69

Dopita, M.A., Evans, I.N. 1986, ApJ, 307, 431

Evans, C. J.\& Howarth, I.D. 2003, MNRAS, 345, 1223

Freedman et al. 2001, ApJ, 553, 47

Gieren, W., Pietrzyński, G., Soszynski, I., Bresolin, F., Kudritzki, R. P., Miniti, D., \& Storm, J. 2005, ApJ, 628,695

Gieren, W. et al. 2005, ESO Messenger, 121, 23

Groth, H. G. 1961, ZAp, 51, 231

Kaufer, A., Venn, K. A., Tolstoy, E., Pinte, C. \& Kudritzki, R. P. 2004, AJ, 127, 2723

Kobulnicky, H.A., Kennicutt, R.C.\& Pizagno, J.L. 1999, ApJ, 514, 544

Kudritzki, R. P. 1973, A\&A, 28, 103

Kudritzki, R.-P., Lennon, D. J. \& Puls, J. 1995, Quantitative spectroscopy of luminous blue stars in distant galaxies, in Proc. ESO Workshop "Science with VLT", Eds. J. P. Welsh \& I. J. Danziger

Kudritzki, R.P., 1998, Quantitative spectroscopy of the brightest blue supergiant stars in galaxies, in "Stellar Physics for the Local Group", eds. A. Aparicio, A. Herrero \&F. Sanchez (Cambridge University Press), p. 149

Kudritzki, R.-P., Puls, J., Lennon, D. J. et al. 1999,A\&A, 350, 970

Kudritzki, R.-P., Bresolin, F., \& Przybilla, N. 2003, ApJ, 582, L83

Kudritzki, R.-P., Urbaneja, M.A., Bresolin, F., Przybilla, N., Gieren, W. \& Pietrzynski, G. 2008, ApJ, submitted

McCarthy, J. K., Lennon, D. J., Venn, K. A., Kudritzki, R. P., Puls, J. \& Najarro, F. 1995, ApJ, 455, L135

McCarthy, J. K., Kudritzki, R. P., Lennon, D. J., Venn, K.A. \& Puls, J. 1997, ApJ, 482, 757

Meynet, \& Maeder, A. 2005, A\&A, 429, 581

Pettini, M. \& Pagel, B.E.J. 2004, MNRAS, 348, L59

Pilyugin, L.S. 2001, A\&A, 369, 594

Przybilla, N., Butler, K., Becker, S. R., Kudritzki, R. P. \& Venn, K.A. 2000, A\&A, 359, 1085

Przybilla, N., Butler, K., Becker, S. R., \& Kudritzki, R. P. 2001a, A\&A, 369, 1009

Przybilla, N., Butler, K. \& Kudritzki, R. P. 2001b,A\&A, 379, 936

Przybilla, N. \& Butler, K. 2001, A\&A, 379, 955

Przybilla, N. 2002, thesis, Fakultaet fuer Physik, Ludwig-Maximilian University, Munich

Przybilla, N., Butler, K., Becker, S. R., \& Kudritzki, R. P. 2006, A\&A, 445, 1099

Przybilla, N., Butler, K., \& Kudritzki, R. P. 2008,in “The Metal-Rich Universe”, eds. G. Israelian \& G. Meynet (Cambridge University Press), in press (astro-ph/0611044)

Schiller, F. \& Przybilla, N. 2008, A\&A, in press (astro-ph/0712004D)

Urbaneja, M. A., Herrero, A. J., Bresolin, F., Kudritzki, R. P., Gieren, W., Puls, J., J. K., Przybilla, N., Najarro, F..\& Pietrzynski, G. 2005, ApJ, 622, 877

Venn, K. A. 1995a, ApJS, 99, 659

Venn, K. A. $1995 \mathrm{a}, A p J, 449,839$

Venn, K. A. 1999, ApJ, 518, 405

Venn, K. A., McCarthy, J. K., Lennon, D. J., Przybilla, N., Kudritzki, R. P. \& Lemke, M. 2000, ApJ, 541, 610

Venn, K. A., Lennon, D. J., Kaufer, A., McCarthy, J. K., Przybilla, N., Kudritzki, R. P., Lemke, M., Skillman, E. D. \& Smartt, S. J. 2001, , 547, 765

Venn, K. A., Tolstoy, E. Kaufer, A. et al. 2003, AJ, 126, 1326

Wolf, B. 1971, $A \& A, 10,383$

Wolf, B. 1972, $A \& A, 20,275$

Wolf, B. 1973, $A \& A, 28,335$

Zaritski, D., Kennicutt, R. C. \& Huchra, J.P. 1994, ApJ, 420, 87 


\section{Discussion}

MASSEY: This is absolutely beautiful work! Let me ask one quesition, though! You have described the precision with which you can measure effective temperatures, surface gravities, abundances, etc. Do you have a sense of their systematic accuracy? Your models must be the best possible now, but what might affect the results of the model that you are unsure of?

KUDRITZKI: There is always the possibility of systematic effects caused by the imperfectness of the models and the NLTE radiative transfer. There are several ways how one can try to address those. For the effective temperatures one can use different methods, ionization equilibria and Balmer jumps, for instance. When the results agree, this is a good indication. With our IfA student Ben Granett we have also tried the alternative approach to determine $T_{\text {eff }}$ from the full integral over the complete UV, optical, IR energy distribution and we found good agreement again. You can test gravities with detached eclipsing binaries. This has been done in the most recent paper by Bonanos et al., 2006. For abundances, the rule is to use as many lines as possible, also in different spectral windows, and to look at the scatter. This work has been done very carefully by Przybilla and collaborators (see his poster papers at this conference).

BURBIDGE: I wish to make three points. (1) This is marvellous work. The spectroscopic method of distance determination is very powerful. (2) Don't be convinced by those who claim that the Hubble Constant is well determined. The value obtained by Sandage and Tammann of about 55 $\mathrm{km} / \mathrm{sec} / \mathrm{Mpc}$ is much more likely correct than the value of $71 \mathrm{~km} / \mathrm{sec} / \mathrm{Mpc}$ being claimed. (3) How long will it take to extend the method to the Virgo Cluster?

KUDRITZKI: Many thanks. I do not have a bias towards any value of the Hubble constant. I think that it is important to have as many independent reliable methods as possible. That is the reason why I am convinced that the FGLR-method is really important. It is an independent way to check existing results. This is also the philosphy of the Araucaria-Project, where we compare Cepheid-distances with TRGB-distances and with the FGLR-method. As for the Virgo cluster, this is a real challenge with Keck, but it will be easy with the TMT.

D'ODORICO: With ELTs like the TMT you expect to achieve the maximum angular resolution at IR wavelengths, where you can use AO at best. Do you think it would be possible to do this type of studies of the most luminous supergiant stars based on IR spectra?

KUDRITZKI: A lot of work has been done already on the IR quantitative spectroscopy of supergiants, as for instance presentated by the papers by Paco Najarro and Fabrice Martins at this meeting. Norbert Przybilla has done a lot already on A supergiants. It will certainly be worthwhile to extent this work to the IR, although the B and A supergiants emit less flux in this spectral window. At the same time, I think, it will also be important to push the AO limit as much towards the visual as by any means possible. While this will certainly not be possible for the ELT first light instruments, in is not excluded in the long term. 